stayed off dialysis for the duration of follow-up (5-35 months after dialysis discontinuation). Patients who recovered renal function were more likely to be female, older and to have had lower urea and creatinine levels at dialysis initiation, but these results did not reach statistical significance. Interestingly, the probable cause of renal failure in 5 of the $8(63 \%)$ patients who recovered renal function was atheroembolism or atherosclerotic renovascular disease. One patient had been scheduled to receive a live donor kidney from his sister after 8 months on dialysis; the surgery was cancelled when she changed her mind, but 3 months later the patient recovered sufficient renal function to discontinue dialysis.

The authors conclude that it might be worth considering delaying transplantation for at least 1 year in certain patients with renal failure who are on dialysis, such as those with atheroembolism, primary malignant hypertension or systemic vasculitis.

Original article Siddiqui S et al. (2007) Recovery of renal function after $90 \mathrm{~d}$ on dialysis: implications for transplantation in patients with potentially reversible causes of renal failure. Clin Transplant [doi:10.1111/j.1399-0012.2007.00755.x]

\section{Online clearance is as reliable as ultrasound dilution for vascular access monitoring}

The ultrasound dilution method of access surveillance in hemodialysis patients has not been widely adopted in clinics despite having been validated and having been commercially available for 10 years. One explanation may be the high cost of implementing this method, which requires procurement of an ultrasound machine and hiring or assigning of a dedicated technician.

In a single-center, pilot study, Lacson et al. compared access flow measurements obtained by the ultrasound dilution technique with those obtained by the online clearance method, which measures blood flow by use of real-time conductivity dialysance. Online clearance programs are integral to many hemodialysis machines, and measurements can be performed by any trained member of staff.

Access flow was measured by both methods concurrently within the first $90 \mathrm{~min}$ of hemodialysis treatment in 50 adult patients (54\% with synthetic arteriovenous grafts, $46 \%$ with native arteriovenous fistulas) in an outpatient hemodialysis center. A significant correlation was found between flow measurements obtained via ultrasound dilution and online clearance (0.93; $P<0.0001)$. Substudies demonstrated that both techniques generate reproducible results both within and between hemodialysis sessions $(P<0.0001$ for all correlations). In addition, the numbers of imaging referrals generated by the two techniques were very similar.

The authors conclude that the online clearance technique is a reliable and affordable alternative to the ultrasound dilution method for monitoring access viability.

Original article Lacson E Jr et al. (2008) Comparison of hemodialysis blood access flow rates using online measurement of conductivity dialysance and ultrasound dilution. Am J Kidney Dis 51: 99-106

\section{Age matching elderly kidney donors and recipients}

The number of elderly patients who need a kidney transplant is increasing, and more organs from elderly donors are being used. The Eurotransplant Senior Program (ESP) was established in seven countries in January 1999 to prospectively match the life expectancy of organs from donors aged $\geq 65$ years with that of recipients in the same age range. Organ allocation in the ESP scheme is also directed by blood type, waiting time, and panel reactive antibody level $<5 \%$ - human leukocyte antigen matching is not undertaken.

Frei et al. compared the outcomes of 1,406 elderly recipients allocated organs via the ESP (mean donor age 70.2 years) with those of two groups allocated kidneys via the standard Eurotransplant Kidney Allocation System: the first group ('old-to-any') included donors aged $\geq 65$ years and recipients of any age $(n=446)$; the second ('any-to-old') included recipients aged $60-64$ years $(n=1,687)$ and donors of any age (mean 45.1 years).

Fewer incidences of delayed graft function occurred in the ESP group ( $P=0.03$ vs the oldto-any group), but the rate of acute rejection was $5-9 \%$ higher. At 5 years, graft and patient survival in the ESP group-at $47 \%$ and $60 \%$, respectively-were poorer than those of the any-to-old cohort, but equivalent to those of the old-to-any group. 